\title{
Effects of fresh gas velocity and thermal expansion on the structure of a Bunsen flame tip
}

\author{
F.J. Higuera \\ E. T. S. Ingenieros Aeronáuticos, UPM, Pza. Cardenal Cisneros 3, 28040 Madrid, Spain
}

\section{A R T I C L E I N F O}

Keywords:

Premixed flames

Bunsen flames

Numerical and asymptotic analysis

\begin{abstract}
A B S T R A C T
Numerical computations and order-of-magnitude estimates are used to describe the tip region of a Bunsen flame where the flame departs from a planar flame at an angle to the incoming fresh gas flow. A single irreversible Arrhenius reaction with high activation energy is assumed. The well-known linear relation between flame velocity and curvature is recovered in the thermodiffusive limit, when the thermal expansion of the gas is left out, for velocities of the fresh gas $\left(U_{0}\right)$ only slightly larger than the velocity of a planar flame $\left(U_{L}\right)$, provided this flame is stable. For large values of the velocity ratio $U_{0} / U_{I}$, the tip region becomes slender and the curvature of the reaction sheet at the tip increases proportionally to $U_{0} / U_{L}$. The thermal expansion of the gas across the flame reduces the aspect ratio of the tip region. A qualitative analysis of the structure of the tip region for very exothermic reactions shows that this region ceases to be slender when the burnt-to-fresh gas temperature ratio becomes of the order of the velocity ratio $U_{0} / U_{t}$. For even larger values of the temperature ratio, the tip region becomes a cap of characteristic size not very different from the thickness of a planar flame.
\end{abstract}

\section{Introduction}

The tip of a Bunsen flame provides a convenient bench in which the effects of curvature and strain on the structure and burning velocity of a premixed flame can be studied. In a comprehensive investigation of Bunsen flames, Lewis and von Elbe [1,2] determined, among other things, the distribution of burning velocity, showing that it is uniform on most of the flame and increases in a region around its tip. Lewis and von Elbe qualitatively explained this result in terms of the convergence of the heat flux from the reaction region to the unburnt gas, on the implicit assumption that this effect dominates that of the divergence of the diffusion flux of the deficient reactant. Sivashinsky $[3,4]$ analyzed the problem in the framework of high activation energy asymptotics, using a thermodiffusive model in which the effect of the thermal expansion of the gas is omitted and the velocity of the gas is uniform. Assuming that the difference $L e-1$, where $L e$ is the Lewis number of the deficient reactant, is of $O(1)$ when the activation energy of the reaction is infinitely large, this author finds that the curvature radius of the thin reaction layer at the tip is large compared to the thickness of the flame, and that the tip is closed for $L e>1$ and open for $L e<1$. In the first case the burning rate increases on approaching the tip, which allows the flame to stand in a flow whose velocity is considerably higher than the velocity of a planar flame. In the second case the burning rate decreases and the flame cannot reach the centerline in a regular manner. The assumption on the Lewis number places this analysis in the realm of slowly varying flames [5], which have been deemed inappropriate for many reactants. Buckmaster [6] adopted the more realistic assumption that the difference Le - 1 is small of the order of the inverse of the dimensionless activation energy, and further simplified the analysis assuming that the velocity of the gas is large compared to the velocity of a planar flame. In these conditions, the flame is slender in a region around the tip where streamwise conduction is negligible and the balance of convection and conduction normal to the flow keeps the burning rate of the same order as for a planar flame. In agreement with experiments, this analysis predicts a closed tip unless the Lewis number is significantly less than unity. Buckmaster and Crowley [7] extended this analysis to take into account the thermal expansion of the gas, showing that the expansion causes the streamlines to diverge and decreases the aspect ratio of the slender tip region.

The effects of a small curvature and a small rate of strain (by the flow of fresh gas) on the local velocity of a stationary flame relative to the fresh gas have been much studied. Following the proposal of Markstein [8], asymptotic analyses for weakly curved and strained flames [9-11] have shown that the variation of the velocity of the flame about that of a planar unstrained flame, $U_{L}$, is proportional to the flame stretch, which is the sum of the strain rate of the flow and the curvature of the flame multiplied by $U_{L}$. The proportionality constant is the Markstein length, which is a function of the properties of the mixture that can be computed from these analy- 
ses or determined experimentally by simultaneously measuring the flame velocity and stretch. Davis et al. [12,13] (see also references in these papers) discuss the effects of the finite thickness of a real flame and the definition of flame velocity on the differences in Markstein length measured in different experiments.

The linear relation between flame velocity and stretch is not expected to be valid at the tip of a Bunsen flame when the curvature of the flame is not small compared to the inverse of its thickness. In an analysis of strongly curved flames carried out using the thermodiffusive model with an artificial velocity field for which an analytic solution exists, Mikolaitis [14,15] found that a conveniently defined flame velocity depends separately and nonlinearly on the strain rate and the curvature of the flame. Echekki and Mungal [16] and Poinsot et al. [17] proposed a heuristic modification of the definition of flame stretch in which the factor $U_{L}$ multiplying the curvature of the flame is replaced by the velocity of the curved and strained flame, and found that the modified relation between velocity, curvature and strain rate, which is no longer linear, compares well with their numerical and experimental results for flame tips of large curvature.

This paper is devoted to the analysis of the tip of a Bunsen flame, both in the thermodiffusive limit and in the case when the thermal expansion is taken into account but the velocity of the fresh gas, $U_{0}$, is large compared to the velocity $U_{L}$ of a planar flame. In both cases, the size of the tip region where the structure of the flame departs from that of a planar flame inclined to the flow is small compared to the total size of the flame, making a local analysis of the tip region possible. A single irreversible Arrhenius reaction with high activation energy is assumed. As was to be expected, the linear relation between flame velocity and curvature discussed above is recovered at the tip of a Bunsen flame in the thermodiffusive limit when $U_{0}$ is only slightly larger than $U_{L}$, and the numerical computations give then values of the Markstein length in good agreement with theory [19]. When the ratio $U_{0} / U_{L}$ is large, the radius of curvature of the reaction layer at the tip becomes of the order of the thickness of the flame or smaller than it. In these conditions, when the thermal expansion of the gas increases, the tip region evolves from a slender region of decreasing aspect ratio, as in the analyses of Buckmaster [6] and Buckmaster and Crowley [7] for null and moderate expansion, to a round cap, apparently as in the experimental results of Mungal and coworkers [16-18].

\section{Formulation}

A uniform stream of a flammable gas mixture issues with velocity $U_{0}$, density $\rho_{u}$ and temperature $T_{u}$ from a circular nozzle into a stagnant inert atmosphere at pressure $p_{a}$. The mixture burns in a stationary axisymmetric flame attached to the rim of the nozzle. A single irreversible exothermic Arrhenius reaction is assumed with a rate $w=\rho B Y \exp (-E / R T)$, where $B$ and $E$ are the frequency factor and activation energy of the reaction, $\rho$ and $T$ are the density and temperature of the gas, and $Y$ is the mass fraction of the reactant that is fully depleted by the flame (the fuel in what follows), for which $Y=Y_{u}$ in the fresh gas. The adiabatic temperature of the flame is $T_{b}=T_{u}+q Y_{u} / c_{p}$, where $q$ is the heat released by the reaction per unit mass of fuel consumed and $c_{p}$ is the specific heat of the gas at constant pressure, which is assumed to be a constant. The Zeldovich number $\beta=E\left(T_{b}-T_{u}\right) / R T_{b}^{2}$ is assumed to be large. A classical high activation energy analysis for $\beta \rightarrow \infty$ gives the velocity and thickness of a planar flame propagating in this mixture as [20]

$U_{L}=\left(2 D_{b} B\right)^{1 / 2} L e \frac{T_{u} / T_{b}}{\beta} \exp \left(-\frac{E}{2 R T_{b}}\right) \quad$ and $\quad \delta_{L}=\frac{\lambda_{u}}{\rho_{u} U_{L} c_{p}}$,

where $D(T)$ and $\lambda(T)$ are the diffusivity of the fuel and the thermal conductivity of the gas, with $\lambda_{u}=\lambda\left(T_{u}\right)$ and $D_{b}=D\left(T_{b}\right)$, and
$L e=\lambda / c_{p} \rho D$ is the Lewis number, which is assumed to be constant. Furthermore, in what follows the Lewis number is assumed to be $L e=1+l e / \beta$ with $l e=O(1)$. Analysis $[9,10,19,20]$ shows that the flame, either planar or curve, consists then of a transport region of characteristic thickness $\delta_{L}$ and a thinner reaction region, of characteristic thickness $\delta_{L} / \beta$, which is not affected by the local curvature and can be treated as a sheet at which the jump conditions (9) below are satisfied.

Scaling distances and velocities with $\delta_{L}$ and $U_{L}$, the density and thermal conductivity of the gas with $\rho_{u}$ and $\lambda_{u}$, the fuel mass fraction with $Y_{u}$, and the pressure referred to $p_{a}$ with $\rho_{u} U_{L}^{2}$, and defining the variables

$\theta=\frac{T-T_{u}}{T_{b}-T_{u}} \quad$ and $\quad H=\beta(\theta+Y-1)$,

which are of order unity across the flame, the governing equations take the form, at leading order in an asymptotic expansion for $\beta \rightarrow \infty$,

$\nabla \cdot(\rho \boldsymbol{v})=0$,

$\rho \boldsymbol{v} \cdot \nabla \boldsymbol{v}=-\nabla p+\operatorname{Pr} \nabla \cdot \tau^{\prime}$

$\rho(1+\gamma \theta)=1$,

$\rho \boldsymbol{v} \cdot \nabla \theta=\nabla \cdot(\lambda \nabla \theta)$,

$\rho \boldsymbol{v} \cdot \nabla H=\nabla \cdot(\lambda \nabla H)+l e \nabla \cdot(\lambda \nabla \theta)$,

$\lambda=(1+\gamma \theta)^{\kappa}$,

outside the reaction sheet. Here the same symbols are used for the dimensionless variables and their dimensional counterparts, which will no longer appear in the paper. The reaction sheet, which is a free surface, is sought in the form $x=h(r)$ or, alternatively, $r=r_{b}(x)$, where $x$ and $r$ are dimensionless distances along the symmetry axis and normal to it. The jump conditions for the variables $\theta$ and $H$ at the reaction sheet are $[9,10,19,20]$

$\theta=1, \quad[|H|]=0,\left.\quad \lambda_{b} \frac{\partial \theta}{\partial n}\right|_{0^{-}}=\frac{\lambda_{b}}{l e}\left[\left|\frac{\partial H}{\partial n}\right|\right]=e^{H / 2}$,

where $[|\cdot|]=(\cdot)_{0^{+}}-(\cdot)_{0}$ and $n$ is the distance normal to the reaction sheet, with $n>0$ in the burnt gas. The jump conditions for the remaining variables follow from (9) and the governing Eqs. (3) and (4) written in conservation form; see, e.g., Ref. [7]. Notice that $\theta=1$ in the burnt gas behind a closed tip.

Eqs. (3)-(5) are the continuity equation, the momentum equation, and the equation of state, in the quasi-isobaric low Mach number approximation. Here $\tau^{\prime}=\mu\left[\nabla \boldsymbol{v}+(\mathbf{\nabla v})^{T}\right]$ is the non-spherical part of the viscous stress tensor, with the viscosity of the gas $\mu$ scaled with its value in the cold gas, $\mu_{u}$. The contribution of the spherical part of the viscous stress tensor is included in the pressure term. Eqs. (6) and (7) are the energy equation and the conservation equation for the excess of enthalpy variable $H$, which is obtained by linearly combining the conservation equations for the energy and the mass of fuel. Eq. (8) gives the power law dependence of the dimensionless conductivity of the gas on temperature that will be adopted in what follows. Since the Prandtl number will be assumed to be constant, the dimensionless viscosity of the gas follows the same law: $\mu(\theta)=\lambda(\theta)$.

The dimensionless parameters that appear in the formulation of the problem are

$\operatorname{Pr}=\frac{\mu_{u} c_{p}}{\lambda_{u}}, \quad l e=\beta(L e-1), \quad \gamma=\frac{T_{b}-T_{u}}{T_{u}}, \quad U=\frac{U_{0}}{U_{L}}$,

which are the Prandtl number of the gas, a reduced Lewis number, a dimensionless measure of the exothermicity of the reaction, and the ratio of the velocity of the stream issuing from the nozzle to the velocity of a planar flame. This latter parameter does not appear 
in (3)-(9) but will enter the problem through the upstream boundary conditions, to be discussed below.

The radius of the nozzle through which the fresh gas is injected is large compared to the thickness of the flame, so that the whole flame appears as a surface of discontinuity at the scale of the nozzle. The thermal expansion of the gas deflects the flow and curves the meridional section of the flame. If $U=O(1)$, then the radius of curvature of the section of the flame is everywhere of the order of the radius of the nozzle, but when $U \gg 1$ the flame is nearly a cone of semiangle $U^{-1}$ except in a small region around its tip where its internal structure departs from that of a planar flame inclined to the flow. In the thermodiffusive limit $\gamma \rightarrow 0$, in which the thermal expansion of the gas is left out, the velocity of the gas is uniform and the flame is a cone with a rounded tip even for $U=O(1)$. This paper is devoted to the analysis of the small region around the tip of the flame for $U \gg 1$ and any $\gamma$, and for $\gamma \rightarrow 0$. It builds on the previous analyses of Buckmaster [6] and Buckmaster and Cowley [7].

An analysis of the flame and the flow away from the tip for large values of $U$ has been carried out elsewhere [21]. This analysis shows that the flame tends to a slender cone and that the flow downstream of the flame approaches that of a uniform axial stream plus a distribution of sources along the axis of the flame, with a strength proportional to the distance to the apparent tip. These results are used here to set up approximate far field boundary conditions expressing the matching of the region around the flame tip with the region away from the tip. For this purpose, a computational domain in the shape of a large cylinder surrounding the flame tip is defined, with the axial distance $x$ measured from the intersection of the reaction sheet with the lateral surface of the cylinder, whose radius is $R_{\infty} \gg 1$ and whose bases are at $x=x_{ \pm \infty}$. The continuity and momentum Eqs. (3) and (4) are rewritten in the equivalent vorticity-stream function formulation. A uniform cold flow is imposed at the inlet base of the cylinder, upstream of the flame,

$\Psi=\frac{1}{2} U r^{2} \quad$ and $\quad \omega=\theta=H=0$ at $x=x_{-\infty}$,

where $\Psi$ and $\omega$ are the stream function and the (azimuthal) vorticity. The temperature, excess of enthalpy and velocity at the lateral boundary upstream of the reaction sheet are those of a planar flame inclined an angle $\arcsin U^{-1}$ to the $x$-axis:

$$
\left.\begin{array}{ll}
\theta=\theta_{L}(n), & H=H_{L}(n), \\
\boldsymbol{v} \cdot \boldsymbol{n}=\frac{1}{1+\gamma \theta_{L}(\boldsymbol{n})}, & |\boldsymbol{v} \times \boldsymbol{n}|=\sqrt{U^{2}-1},
\end{array}\right\} \text { at } r=R_{\infty}, x<0,
$$

where $\boldsymbol{n}$ is a unit vector normal to the reaction sheet, $n=x / U$, and the two functions $\theta_{L}(n)$ and $H_{L}(n)$ are the distributions of temperature and excess of enthalpy in the transport region of a planar flame; see, e.g., Ref. [20]. The outlet conditions

$\theta=1, \quad \frac{\partial H}{\partial n_{\infty}}=\frac{\partial(r \omega)}{\partial n_{\infty}}=0, \quad \Psi \sim \Psi_{f f}$,

are used in the rest of the boundary, at $r=R_{\infty}, x>0$ and at $x=x_{+\infty}$. Here $\Psi_{f f}$ is the stream function of the far field flow discussed above and $\partial / \partial n_{\infty}$ denotes the derivative normal to the boundary.

For the numerical treatment, the free boundary problem (3)(13) is rewritten in terms of the variables $(\xi, r)$ with $\xi=\left(x-x_{-\infty}\right) /\left[h(r)-x_{-\infty}\right]$, which maps the reaction sheet onto $\xi=1$, discretized using finite differences, and solved with a standard iterative method. This transformation is valid only for closed, non-bulging flames, to which attention is restricted in what follows. No tendency for $h(r)$ to develop an infinite slope, which would mark the beginning of bulging (see Refs. [6,7]), was observed in the computations that have been carried out.

\section{Results and discussion}

\subsection{Thermodiffusive limit}

Problem (3)-(13) simplifies in the thermodiffusive limit $\gamma \rightarrow 0$, in which the density and thermal conductivity of the gas are constant, $\rho=\lambda=1$, and its velocity and pressure are $\boldsymbol{v}=U \boldsymbol{i}$ and $p=0$, where $\boldsymbol{i}$ is a unit vector in the direction of the symmetry axis. The problem reduces then to the transport Eqs. (6) and (7) for the temperature and excess of enthalpy, with the conditions (9) at the reaction sheet and (11)-(13) in the far field. The solution depends on the parameters $U$ and $l e$.

Fig. 1 shows the meridional section of the reaction sheet computed from the numerical solution of this problem for different values of $U$ and le. Fig. 2 shows the distribution of burning rate, $\partial \theta /\left.\partial n\right|_{0}$, for these solutions. The height of the flame increases with $U$, as was to be expected, and decreases when le increases, which is due to the increase of the burning rate around the tip with le. Fig. 2 also displays the well-known result that the burning rate is uniform and equal to that of a planar flame (unity in dimensionless variables) when $l e=0$. This is because the solution of Eq. (7) with the boundary conditions of the problem is then $H=0$, to be carried to the right-hand side of the last condition (9).

Fig. 3 shows the distributions of temperature (at the left-hand side of each panel) and excess of enthalpy for two values of $U$ and le. Away from the tip, where the curvature of the reaction sheet is small, the profiles of these variables are similar to those of a planar flame, which for a medium with $\rho=\lambda=1$ are $\theta_{L}=\exp (n), H_{L}=$ le $n \exp (n)$ for $n<0$ and $\theta_{L}=1, H_{L}=0$ for $n>0$, where $n$ is again the distance normal to the reaction sheet. When the curvature of the reaction sheet increases, conduction parallel to this sheet comes into play in the transport region, so that the thickness of the temperature and excess of enthalpy profiles increases, or at least does not decrease in inverse proportion to the velocity, as it would do if the transport region were planar. This allows the flame tip to stand in front of a fast incoming flow with only a moderate increase of the burning rate $\partial \theta /\left.\partial n\right|_{0}$, and even to cope with the decrease of the burning rate that occurs when $l e<0$. The excess of enthalpy has a maximum or a minimum ahead of the reaction sheet, depending on the sign of $l e$. This is due to the source term in the right-hand side of Eq. (7), which reflects

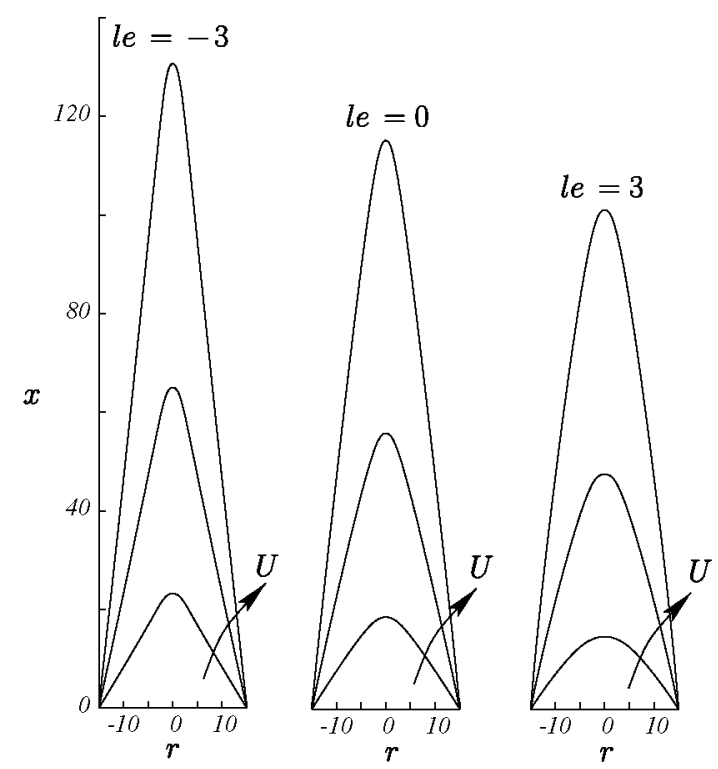

Fig. 1. Meridional section of the reaction sheet for $\gamma=0$, three values of $l e$, and $U=2,5$ and 10 , increasing as indicated by the arrows. 


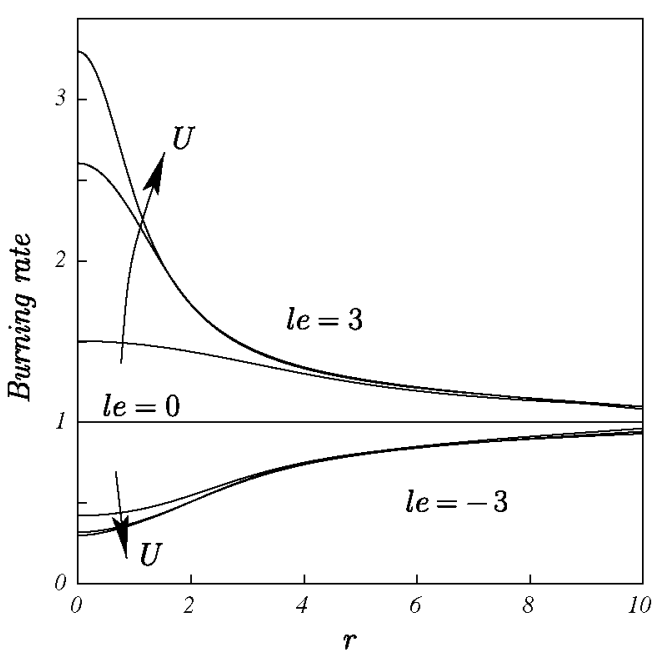

Fig. 2. Dimensionless burning rate along the reaction sheet for $\gamma=0, l e=3$ (upper three curves), 0 (middle straight line), and -3 (lower three curves), and $U=2,5$ and 10 , increasing as indicated by the arrows.

the small difference between heat and mass diffusivities. The extreme of $H$ increases with the curvature of the flame, and the excess or defect of enthalpy extends to the reaction sheet. This explain the variation of the burning rate at the tip and the subsequent changes of the height and curvature of the reaction sheet, in order for transverse conduction to thicken the temperature distribution at the tip as le decreases.

A normal velocity of propagation of a premixed flame relative to the fresh gas can still be defined when the flame is slightly curved or the velocity of the gas is not strictly uniform. It has been shown in Refs. [9-11,22,23] that when the curvature of the reaction sheet is small compared to the inverse of the flame thickness, and the velocity gradient in the fresh gas ahead of the flame is small compared to the residence time of the gas in the flame, the normal velocity of the flame is, in the dimensionless variables used here,

$u_{n}=1+\operatorname{Ma}(\boldsymbol{\nabla} \cdot \boldsymbol{n}+\boldsymbol{n} \cdot \nabla \boldsymbol{v} \cdot \boldsymbol{n})$,

where $\mathbf{n}$ is the normal to the reaction sheet, the strain rate $\boldsymbol{n} \cdot \nabla \boldsymbol{v} \cdot \boldsymbol{n}$ is evaluated immediately upstream of the flame, and $M a$ is the Markstein number. This quantity fully characterizes the response of the flame to small curvature and strain. It was first introduced by Markstein [8] as a phenomenological constant and has been subsequently computed using asymptotic methods for small values of the quantity in parentheses in (14). For the case of a single overall Arrhenius reaction, it is $[9-11,24]$

$M a=(1+\gamma) \frac{(1+\gamma)^{\kappa}-1}{\kappa \gamma}+I l e$,

with $\quad I=-\frac{1}{2} \int_{0}^{1}(1+\gamma \theta)^{\kappa-1} \ln \theta \mathrm{d} \theta$,

which in the thermodiffusive limit $\gamma \rightarrow 0$ becomes $M a=\frac{1}{2}(l e+2)$; see also Ref. [19]. In the uniform gas flow considered here, $\boldsymbol{n} \cdot \nabla \boldsymbol{v} \cdot \boldsymbol{n}=\mathbf{0}$ and the variation of the flame velocity is proportional to twice the mean curvature of the reaction sheet $\boldsymbol{\nabla} \cdot \boldsymbol{n}$.

The ratio $(U-1) / \nabla \cdot \boldsymbol{n}$ evaluated at the tip of the flame is shown in Fig. 4 as a function of $U$. Numerical solutions for small values of $U-1$ have been computed for $l e>-2$, in which case the curvature of the flame is small and the ratio $(U-1) / \nabla \cdot \boldsymbol{n}$ tends to $(l e+2) / 2$, in agreement with the established theory [19]. The numerical method failed to converge to a stationary solution for small values of $U-1$ and $l e<-2$, probably reflecting the fact that the planar flame is unstable to long wavelength perturbations when
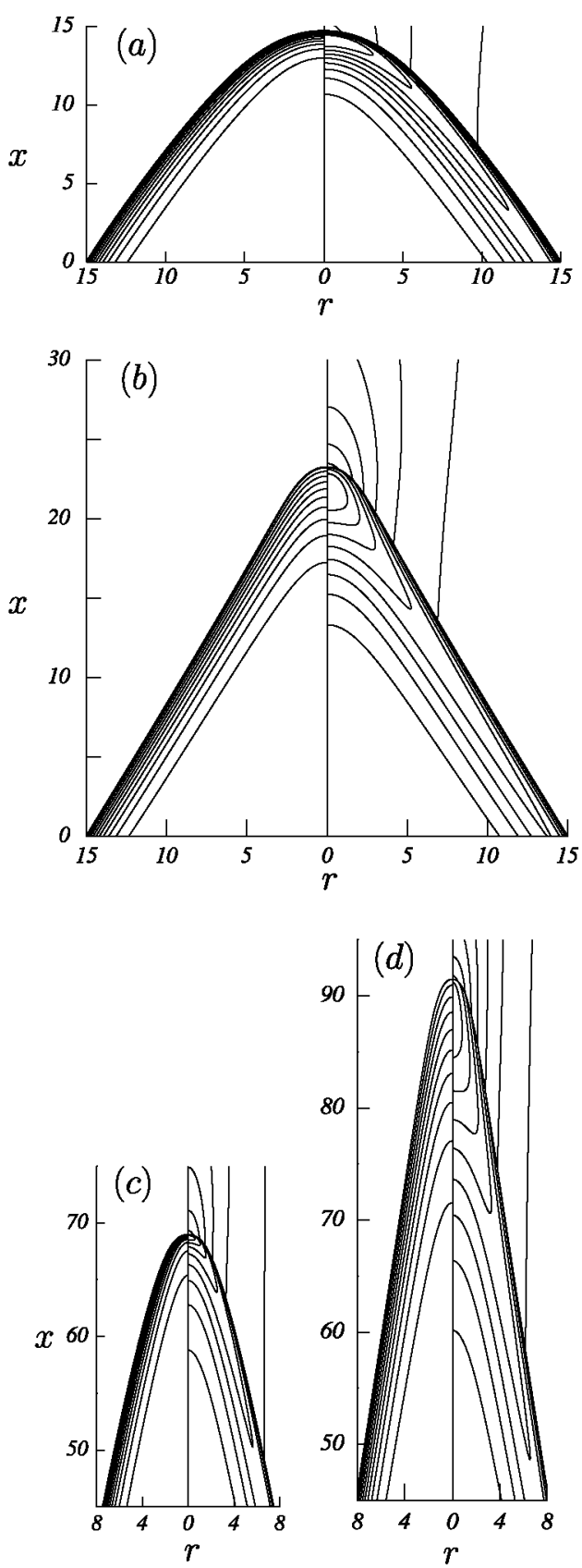

Fig. 3. Distributions of $\theta$ (left-hand side of each panel) and $H$ for (a) $l e=3, U=2(\max H=1.77) ; \quad$ (b) $\quad l e=-3, U=2(\min H=-2.47) ; \quad$ (c) $l e=3, U=7(\max H=3.06)$; and (d) $l e=-3, U=7(\min H=-2.78)$. In all the cases $\gamma=0$. The computations have been carried out with $R_{\infty}=15$.

le $<-2$; see Ref. [19]. When $U$ increases, the ratio $(U-1) / \boldsymbol{\nabla} \cdot \boldsymbol{n}$ increases, but it seems to approach a finite asymptotic value for large values of $U$

Buckmaster [6] showed that axial diffusion is negligible almost everywhere when $U \gg 1$ and computed the solution of the parabolic problem that is obtained in the limit $U \rightarrow \infty$. The characteristic distance to the tip at which the transport region of the flame fills the cross-section of the reaction sheet and the temperature at the symmetry axis begins to increase is $\Delta x_{0}=U$. This result follows from an order-of-magnitude balance of axial convection, $U \partial \theta / \partial x \sim U / \Delta x_{0}$, and radial conduction, $r^{-1} \partial(r \partial \theta / \partial r) / \partial r \sim 1$, in the energy Eq. (6), where use has been made of the condition that $(\theta, r)=O(1)$ in the transport region, in order for the dimensionless 


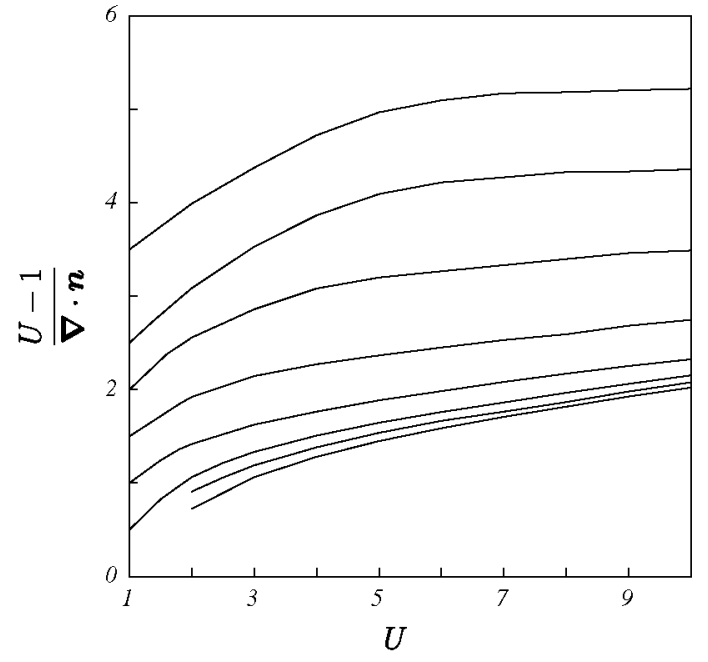

Fig. 4. Value of $(U-\mathbf{1}) / \boldsymbol{\nabla} \cdot \boldsymbol{n}$ at the tip of the flame as a function of $U$ for $\gamma=0$ and $l e=-5,-2,-1,0,1,2,3$ and 5 , increasing from bottom to top.

burning rate $\partial \theta /\left.\partial n\right|_{0} \approx \partial \theta /\left.\partial r\right|_{r_{b}}$ to remain of $O(1)$ for a slender flame. At distances from the tip $\Delta x \ll \Delta x_{0}$, the radius of the reaction sheet is $r_{b}(x) \ll 1$ and the convection-conduction balance reads $U \Delta \theta / \Delta x \sim \Delta \theta / r_{b}^{2}$, where $\Delta \theta \sim r_{b}$ to keep $\partial \theta / \partial r=O(1)$. This balance gives $r_{b} \sim(\Delta x / U)^{1 / 2}$, until axial conduction comes into play when $\left(\Delta x, r_{b}\right) \sim U^{-1}$ and causes the final round off of the reaction sheet with $\partial \theta / \partial x=O(1)$ at its tip. These estimates suggest that the curvature of the tip scales as $U$, in line with the trends of the numerical results in Fig. 4 for large $U$.

\subsection{Effect of the gas expansion}

The far field boundary conditions discussed in Section 2 are valid only for large values of $U$ when $\gamma>0$. There is no reason to expect a localized flame tip region when $U$ is not large; the radius of curvature of the meridional section of the reaction sheet is then of the order of the radius of the nozzle everywhere on the flame.

Fig. 5 shows the reaction sheet computed for $U=5$ and different values of $\gamma$. The effect of the gas expansion on the temperature distribution can be seen in Fig. 6 for a sample case, while Fig. 7 illustrates the deflection of the streamlines and the generation of vorticity at the flame that accompany the expansion of the gas. The decrease of the height of the flame and the curvature of its tip when the expansion of the gas increases was first noticed by Buckmaster and Cowley [7], who analyzed the problem in the asymptotic limit $U \rightarrow \infty$ with $\gamma=O(1)$.

The scaling discussed at the end of Section 3.1 should be valid in this limit and predicts that the curvature at the tip increases proportionally to $U$. At variance with this prediction, the ratio $(U-1) / \nabla \cdot \boldsymbol{n}$ evaluated at the tip of the reaction region from the numerical solution of (3)-(13) (Fig. 8) does not level off for large $U$, and even seems to increase linearly with $U$ at the largest values of the thermal expansion $\gamma$ for which a solution has been computed. Such linear increase would mean that the curvature $\boldsymbol{\nabla} \cdot \boldsymbol{n}$ tends to a constant value for large $U$, a result suggested by Echekki and Mungal [16] on the basis of their experiments with a slot burner and later rationalized by Poinsot et al. [17] from a heuristic extension of the result (14).

This discrepancy seems to imply that the asymptotic limit $U \rightarrow \infty$ with $\gamma=O(1)$ does not give a faithful representation of the conditions of the flow in the experiments in Ref. [16] and the present computations, where $\gamma$ is in fact of the order of $U$ or larger than it. An analysis of the problem for large values of both $\gamma$ and $U$ would be of interest and is outlined in what follows.

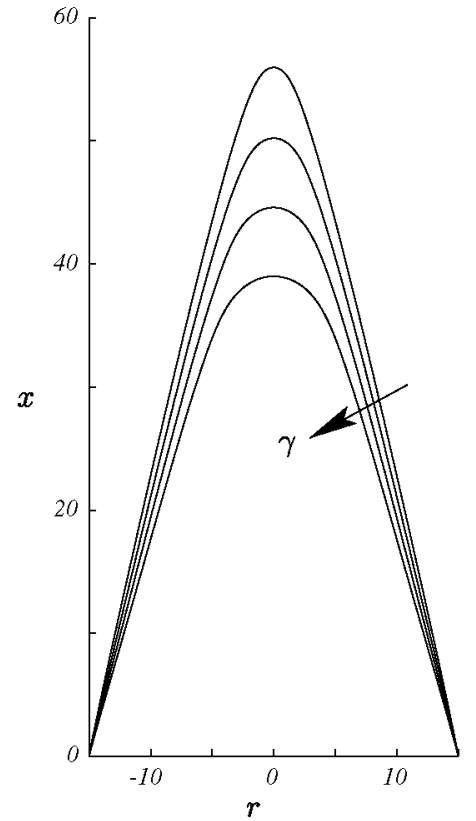

Fig. 5. Meridional section of the reaction sheet for $\operatorname{Pr}=1, l e=0, U=5$, and $\gamma=0$, 1,3 and 7 , increasing as indicated by the arrow.
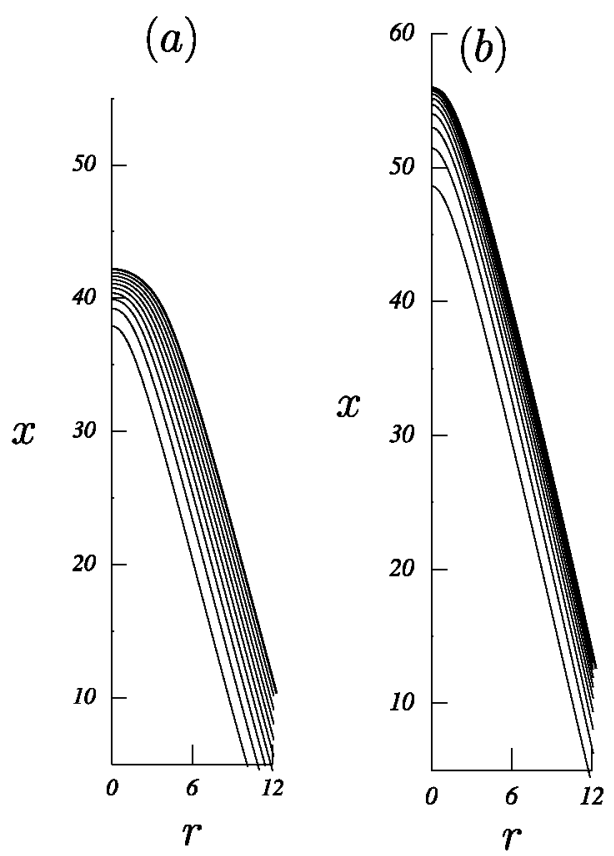

Fig. 6. Distribution of $\theta$ around the tip of the flame for $P r=1, l e=0, U=5$, and $\gamma=5$ [in (a)] and 0 [in (b)]. Computations carried out with $R_{\infty}=15$.

Fig. 6 shows that the thickness of the temperature distribution increases with $\gamma$ away from the tip and decreases at the tip. The second effect will be discussed later. The first one is due to the increase of the dimensionless conductivity of the gas with temperature. When $\gamma \gg 1$, the conductivity (8) can be approximated by $(\gamma \theta)^{\kappa}$ in most of the transport region, with the exception of a low temperature tail of order unity thickness where $\theta=O\left(\gamma^{-1}\right)$. For a planar flame, the transport Eqs. (6) and (7) take the form $\partial \theta_{L} / \partial n=\partial\left[\left(\gamma \theta_{L}\right)^{\kappa} \partial \theta_{L} / \partial n\right] / \partial n$ and $\partial H_{L} / \partial n=\partial\left[\left(\gamma \theta_{L}\right)^{\kappa} \partial\left(H_{L}+l e \theta_{L}\right) /\right.$ $\partial n] / \partial n$. Their solution with the conditions $\theta_{L}=H_{L}=0$ in the fresh gas and $\theta_{L}-1=H_{L}=0$ at the reaction sheet $n=0$ is 


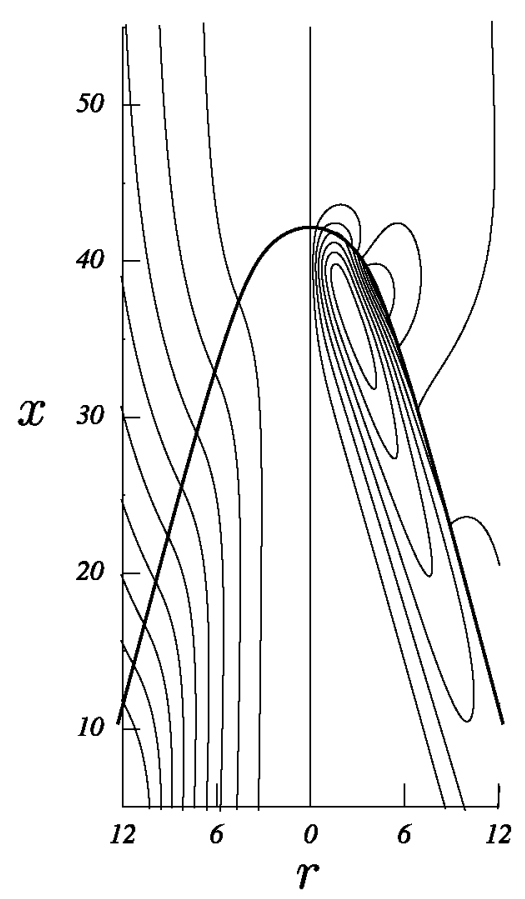

Fig. 7. Streamlines (left-hand side; 10 equispaced contours of $\Psi$ between 0 and 312 ) and vorticity for $\operatorname{Pr}=1, l e=0, \gamma=5$ and $U=5$ ( $\max \omega=0.128)$. Computations carried out with $R_{\infty}=15$.

$\theta_{L}=\left(1+\kappa n / \gamma^{\kappa}\right)^{1 / \kappa}$ and $H_{L}=-l e \theta_{L} \ln \theta_{L}$. Thus, leaving out the low temperature tail mentioned above, $\theta_{L}$ falls to zero algebraically at the finite dimensionless distance $\delta=\gamma^{\kappa} / \kappa$ upstream of the reaction sheet.

This compact transport region allows a simplified treatment of the region of the flame where the radius of the cross-section of the reaction sheet, $r_{b}(x)$, is of order $\delta$ but the transport region still does not extend to the symmetry axis. Dimensionless pressure variations of order $\gamma$ are expected in the transport region and in the burnt gas, where the dimensionless density is of order $\gamma^{-1}$ and dimensionless velocity variations of order $\gamma$ must exist to reorient the stream that crosses the flame; see, e.g., Ref. [21] for details. However, these pressure variations will not induce important changes in the stream of cold gas insofar as $U^{2} \gg \gamma$. The dimensionless mass flux of cold gas entering the region of interest is therefore of order $U \delta^{2}$. The characteristic axial length of this region, say $\Delta x_{1}$, can be estimated from the condition that this mass flux must be consumed in an area of $O\left(\Delta x_{1} \delta\right)$ of a flame whose dimensionless burning rate is of order unity the burning rate of a planar flame). This balance of mass gives $\Delta x_{1}=U \delta$, so that $\mathrm{d} r_{b} / \mathrm{d} x \sim U^{-1} \ll 1$. Leaving out the effect of axial conduction in the right-hand sides of Eqs. (6) and (7), and introducing the variables $(x, \tilde{r})$ with $\tilde{r}=r-r_{b}(x)$, these equations and the continuity Eq. (3) take the form

$$
\begin{aligned}
& \frac{\partial}{\partial x}(\rho \boldsymbol{u})+\frac{1}{r_{b}+\tilde{r}} \frac{\mathrm{d} r_{b}}{\mathrm{~d} x} \rho \boldsymbol{u}+\frac{1}{r_{b}+\tilde{r}} \frac{\partial}{\partial \tilde{r}}\left[\left(r_{b}+\tilde{r}\right) \rho \tilde{v}\right]=0, \\
& \frac{\partial}{\partial x}(\rho u \theta)+\frac{1}{r_{b}+\tilde{r}} \frac{\mathrm{d} r_{b}}{\mathrm{~d} x} \rho u \theta+\frac{1}{r_{b}+\tilde{r}} \frac{\partial}{\partial \tilde{r}}\left[\left(r_{b}+\tilde{r}\right) \rho \tilde{v} \theta\right] \\
& =\frac{1}{r_{b}+\tilde{r}} \frac{\partial}{\partial \tilde{r}}\left[(\gamma \theta)^{\kappa}\left(r_{b}+\tilde{r}\right) \frac{\partial \theta}{\partial \tilde{r}}\right], \\
& \frac{\partial}{\partial x}(\rho u H)+\frac{1}{r_{b}+\tilde{r}} \frac{\mathrm{d} r_{b}}{\mathrm{~d} x} \rho u H+\frac{1}{r_{b}+\tilde{r}} \frac{\partial}{\partial \tilde{r}}\left[\left(r_{b}+\tilde{r}\right) \rho \tilde{v} H\right] \\
& \quad=\frac{1}{r_{b}+\tilde{r}} \frac{\partial}{\partial \tilde{r}}\left[(\gamma \theta)^{\kappa}\left(r_{b}+\tilde{r}\right) \frac{\partial}{\partial \tilde{r}}(H+l e \theta)\right],
\end{aligned}
$$
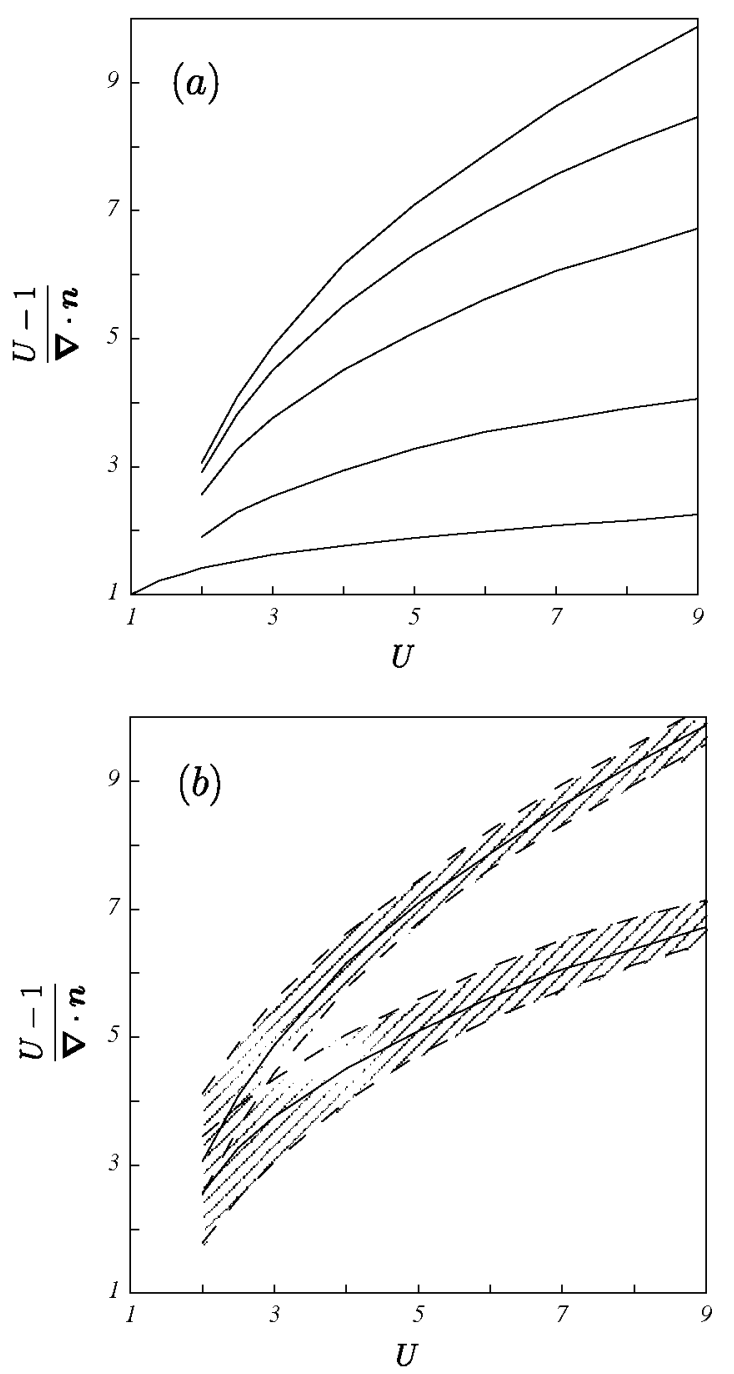

Fig. 8. Value of $(U-1) / \mathbf{V} \cdot \boldsymbol{n}$ at the tip of the flame as a function of $U$ for $\operatorname{Pr}=1$. In (a), $l e=0$ and $\gamma=0,1,3,5$ and 7 , increasing from bottom to top. In (b), the hatched regions are traversed upward when $l e$ increases from -5 to 5 for $\gamma=3$ (lower region) and 7.

where

$\rho \tilde{v}=\rho v-\frac{\mathrm{d} r_{b}}{\mathrm{~d} x} \rho u$

and $(u, v)$ are the axial and radial components of the gas velocity. The first two terms on the left-hand side of each of Eqs. (16)-(18) are of order $1 /(\gamma \delta)$, when use is made of the estimates of $\Delta x_{1}$ and $\mathrm{d} r_{b} / \mathrm{d} x$ worked out above. The right-hand sides of (17) and (18) are much larger, of order $\gamma^{\kappa} / \delta^{2} \sim 1 / \delta$, and must therefore be balanced by the third term on the left-hand sides of these equations. This condition requires that $\rho \tilde{v} \sim 1$. Eqs. (16)-(18) can therefore be simplified leaving out the first two terms on their left-hand sides. Written back in the original variables, the simplified equations are

$$
\begin{aligned}
& \frac{1}{r} \frac{\partial}{\partial r}(r \rho \tilde{v})=0, \\
& \frac{1}{r} \frac{\partial}{\partial r}(r \rho \tilde{v} \theta)=\frac{1}{r} \frac{\partial}{\partial r}\left[(\gamma \theta)^{\kappa} r \frac{\partial \theta}{\partial r}\right], \\
& \frac{1}{r} \frac{\partial}{\partial r}(r \rho \tilde{v} H)=\frac{1}{r} \frac{\partial}{\partial r}\left[(\gamma \theta)^{\kappa} r \frac{\partial}{\partial r}(H+l e \theta)\right],
\end{aligned}
$$

which coincide with the equations for the transport region of a cylindrical flame around a line source of flammable gas. Eq. (20) 
gives $r \rho \tilde{v}=\phi / 2 \pi$, where $\phi$ is the mass flux issuing from the source, which is to be burnt at the flame. The radius $r_{b}$ of the reaction sheet depends on $\phi$, but it is simpler to use $r_{b}$ to parametrize the solution. Thus, the solution of (20)-(22) with the conditions $\theta=H=0$ in the cold gas and $\theta-1=H=0, \gamma^{\kappa} \partial \theta / \partial r=1$ at $r=r_{b}$ is

$\theta=\left(1-\frac{\ln r_{b} / r}{\ln r_{b} / r_{u}}\right)^{1 / \kappa}, \quad H=-l e \theta \ln \theta, \quad r \rho \tilde{v}=\frac{\phi}{2 \pi}=r_{b}$,

where $r_{u}=r_{b} / \exp \left(\gamma^{\kappa} / \kappa r_{b}\right)$ is the radius of the inner boundary of the transport region, where $\theta=0$. Finally, carrying the last result (23) to (19) and particularizing this equation at $r=r_{u}$, where $\rho=1, u=U$, and $v=0$, we find $U \mathrm{~d} r_{b} / \mathrm{d} x=-r_{b} / r_{u}=\exp \left(\gamma^{\kappa} / \kappa r_{b}\right)$, which is an equation for the streamwise evolution of the radius of the reaction sheet.

The temperature distribution in (23) reduces to that of a planar flame $\left[\theta_{L}\right.$ in the paragraph above (16)] and the velocity of the gas normal to the cold boundary of the transport region, $\left.\tilde{v}\right|_{r_{u}}=r_{b} / r_{u}$, tends to the unity when $r_{b} \gg \delta=\gamma^{\kappa} / \kappa$. On the other hand, this velocity increases exponentially, and $r_{u}$ decreases exponentially, when $r_{b}$ becomes small compared to $\delta$. The condition $r_{u} \sim 1$ (the thickness of the cold side tail of the transport region where $\theta \sim \gamma^{-1}$ ) marks the effective disappearance of the the core of cold gas around the symmetry axis (at least for $U \gg \gamma$; see discussion below).

Assuming that the remaining fresh gas is also consumed in a slender region, the characteristic length and radius of the reaction sheet in this region, say $\Delta x_{0}$ and $r_{b 0}$, can be estimated using the same balances as at the end of Section 3.1; namely that convection and radial conduction balance each other in the energy Eq. (6), and that the dimensionless burning rate is of order unity. Taking into account the reduced density and the increased conductivity of the heated gas, these order-of-magnitude balances read now $(U / \gamma) / \Delta x_{0} \sim \gamma^{\kappa} / r_{b 0}^{2}$ and $\gamma^{\kappa} / r_{b 0} \sim 1$, whence $\Delta x_{0} \sim U / \gamma^{1-\kappa}$ and $r_{b 0} \sim \gamma^{\kappa}$. Finally, proceeding as in Section 3.1, it can be seen that axial conduction comes into play at distances from the tip of the order of $\gamma^{1+\kappa} / U$, which is also the order of the curvature radius of the reaction sheet at the tip.

The dimensionless mass of gas burned per unit time in the region of characteristic length $\Delta x_{0}$ is of order $\Delta x_{0} r_{b 0} \sim \gamma^{2 \kappa-1} U$, which coincides with the estimate for the analogous region of Section 3.1 multiplied by a factor $\gamma^{2 k-1}$. The aspect ratio of this region is $\Delta x_{0} / r_{b 0} \sim U / \gamma$. The region ceases to be slender when $\gamma \sim U$, in which case $\left(\Delta x_{0}, r_{b 0}\right) \sim \gamma^{\kappa}$. When $\gamma \gg U$, the slender region is replaced by a round cap similar to that of Fig. $6 a$. The mass flux of fresh gas burned in this cap is large compared to the mass flux of preheated gas left at the end of the preceding region of characteristic length $\Delta x_{1}$, so that most of the gas enters the cap as a narrow stream tube of cold gas. If the cap is approximated by a hemisphere of radius $r_{b}$, the temperature distribution and the burning rate would be those of a spherical flame around a point source of flammable gas, which are given by $\theta=\left[\left(r_{u}^{-1}-r^{-1}\right) /\left(r_{u}^{-1}-r_{b}^{-1}\right)\right]^{1 / \kappa}$ and $\gamma^{\kappa} \partial \theta /\left.\partial \boldsymbol{r}\right|_{r_{b}}=\left(\gamma^{\kappa} / \kappa\right)\left(r_{u} / r_{b}^{2}\right) /\left(1-r_{u} / r_{b}\right)$, where $r$ is now the distance from the point source and $r_{u}$ is the radius of the cold boundary of the transport region. (These results follow from equations similar to (20)-(22) with the factors $r$ replaced by $r^{2}$.) If the radius of the inlet stream tube is assumed to coincide with $r_{u}$ and the burning rate is assumed to be equal to the unity, which is an exact result for $l e=0$, then $\pi U r_{u}^{2}=2 \pi r_{b}^{2}$ and $\gamma^{k} \partial \theta /\left.\partial r\right|_{r_{b}}=1$, whence $r_{b}=\left(\gamma^{\kappa} / \kappa\right) /\left[(U / 2)^{1 / 2}-1\right]$ and $r_{u}=r_{b} /(U / 2)^{1 / 2}$. This $r_{b}$ is formally small compared to the thickness $\delta=\gamma^{\kappa} / \kappa$ of a planar flame, but the factor $(U / 2)^{1 / 2}-1$ in the denominator is not very large for realistic values of $U$ (it is only $1 / 2$ for $U=9$ ).

The description given here simplifies somewhat for the twodimensional flame of a slot burner. The reaction sheet is then a wedge away from the tip, and the flame remains an inclined planar flame until the transport regions adjacent to the two wings of the reaction sheet merge, which happens when the distance between the wings is $2 \delta$. The wedge will end in a round cap of characteristic radius not very different from $\delta$ when $\gamma \gg U$. It is worth noticing that the Markstein number (15) is $M a \approx \delta$ for $\gamma \gg 1$ and any $|l e| \ll \gamma$, which gives some support to the claim of Echekki and Mungal [16] and Poinsot et al. [17].

\section{Conclusions}

A numerical analysis of the tip of a Bunsen flame has been carried out for the case of a single irreversible Arrhenius reaction with high activation energy. The results of Buckmaster [6] for the thermodiffusive limit are extended to values of the velocity of the gas, $U_{0}$, of the order of the velocity of the planar flame, $U_{L}$, though larger than it. When the ratio of these two velocities is near unity and the planar flame is stable, the curvature of the reaction sheet at the tip of the Bunsen flame is proportional to $U_{0} / U_{L}-1$, and the Markstein number determined from this linear relation agrees with the theoretical value. When the planar flame is instable, the numerical computations fail to converge to a stationary Bunsen flame for small values of $U_{0} / U_{L}-1$. The slender flame tip region of Buckmaster [6] is recovered for large values of $U_{0} / U_{L}$. Then the curvature of the reaction sheet at the tip tends to increase linearly with $U_{0} / U_{L}$, but the slope of this asymptote is different from the Markstein number of the flame.

The thermal expansion of the gas across the flame reduces the aspect ratio of the tip region, in agreement with the results of Buckmaster and Cowley [7]. A qualitative analysis for large values of the thermal expansion, measured by the ratio $\gamma$ of the temperature rise across the flame to the temperature of the fresh gas, suggests a two-tiered structure of the tip region. The reaction sheet departs from a cone at distances from the tip of order $\left(U_{0} / U_{L}\right) \delta$, where $\delta$ is the thickness of the planar flame, which increases with $\gamma$. This is a slender region where the flame is affected by the curvature of its cross-section but a core of fresh cold gas still exists around the symmetry axis. It is followed by a second region where the remaining flux of fresh gas is heated up. If $1 \ll \gamma \ll U_{0} / U_{L}$, this second region is also slender and the radius of curvature of the reaction sheet at the tip is of order $\left(\gamma U_{L} / U_{0}\right) \delta$. On the contrary, if $\gamma \gg U_{0} / U_{L}$, the second region is a rounded cap of characteristic size $\delta$ or somewhat smaller than $\delta$. The first of these regions is absent for a two-dimensional flame attached to a slot burner.

\section{Acknowledgments}

This work was supported by the Comunidad de Madrid through Projects COMLIMAMS, S-0505/ENE-229, and HYSYCOMB, P2009/ ENE-1597.

\section{References}

[1] B. Lewis, G. von Elbe, J. Chem. Phys. 11 (1943) 75-97.

[2] B. Lewis, G. von Elbe, Combustion, Flames and Explosions of Gases, Academic Press, New York, 1961 [Chapter 5].

[3] G.I. Sivashinsky, ASME J. Heat Transfer 11 (1974) 530-535.

[4] G.I. Sivashinsky, J. Chem. Phys. 62 (1975) 638-643.

[5] J.D. Buckmaster, G.S.S. Ludford, Theory of Laminar Flames, Cambridge University Press, Cambridge, 1982

[6] J. Buckmaster, Combust. Sci. Technol. 20 (1979) 33-40.

[7] J. Buckmaster, A.B. Crowley, J. Fluid Mech. 131 (1983) 341-361

[8] G.H. Markstein, J. Aerospace Sci. 18 (1951) 199.

[9] P. Clavin, F.A. Williams, J. Fluid Mech. 116 (1982) 251-282

[10] M. Matalon, B.J. Matkowsky, J. Fluid Mech. 124 (1982) 239-259.

[11] P. Clavin, G. Joulin, J. Phys. Lett. 44 (1983) L1-L12.

[12] S.G. Davis, J. Quinard, G. Searby, Combust. Flame 130 (2002) 112-122.

[13] S.G. Davis, J. Quinard, G. Searby, Combust. Flame 130 (2002) 123-136

[14] D.W. Mikolaitis, Combust. Flame 57 (1984) 25-31.

[15] D.W. Mikolaitis, Combust. Flame 58 (1984) 23-29. 
16] T. Echekki, M.G. Mungal, Proc. Combust. Inst. 23 (1990) 455-461.

[17] T. Poinsot, T. Echekki, M.G. Mungal, Combust. Sci. Technol. 81 (1992) 45 73.

[18] M.G. Mungal, L.M. Lourenco, A. Krothapalli, Combust. Sci. Technol. 106 (1995) $239-265$

[19] G.I. Sivashinsky, Ann. Rev. Fluid Mech. 15 (1983) 179-199.
[20] F.A. Williams, Combustion Theory, 2nd ed., Benjamin Cummings, Menlo Park, CA, 1985.

[21] F.J. Higuera, Combust. Flame 156 (2009) 1063-1067.

[22] P. Clavin, Prog. Energy Combust. Sci. 11 (1985) 1-59.

[23] P. Clavin, Proc. Combust. Inst. 28 (2000) 569-585.

[24] P. Clavin, P.L. Garcia-Ybarra, J. Méc. Thêor. Appl. 2 (1983) 245-263. 